\title{
Stable analytic bounce in non-local Einstein-Gauss-Bonnet cosmology
}

\author{
Alexey S. Koshelev \\ Theoretische Natuurkunde, Vrije Universiteit Brussel and The International Solvay Insti- \\ tutes, Pleinlaan 2, B-1050 Brussels, Belgium \\ E-mail: alexey.koshelev@vub.ac.be
}

\begin{abstract}
We consider the most general quadratic in curvature stringy motivated non-local action for the modified Einstein's gravity. We present exact analytic cosmological solutions including the bouncing ones and develop the relevant techniques for the further study of this type of models. We also elaborate on the perturbation formalism and argue that the found bouncing solution is stable during the bounce phase.
\end{abstract}

Keywords: alternatives to inflation, string theory and cosmology, cosmological perturbation theory, modified gravity 


\section{Contents}

1 Introduction $\quad 1$

2 Action and equations of motion $\quad 3$

3 Exact solutions based on the recursion relations 4

3.1 Recursion relations 4

3.2 Exact analytic bounce 5

4 More analytic cosmological solutions? $\quad 8$

4.1 Closely related solutions $\quad 8$

4.2 Model reformulation using $\tilde{G}_{\nu}^{\mu} \quad 9$

$\begin{array}{lll}4.3 & \text { Avoiding recursion relations } & 10\end{array}$

$\begin{array}{lll}4.4 & \text { Other solutions } & 11\end{array}$

5 A road-map to perturbations $\quad 13$

5.1 Bianchi identity 13

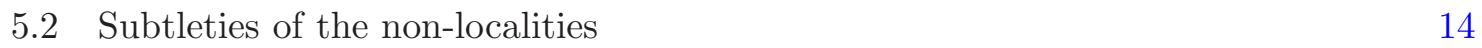

$\begin{array}{ll}5.3 \text { de Sitter limit } & 15\end{array}$

6 Summary and Outlook 16

\section{Introduction}

Recent observations [1] strongly support that primordial inflation is the theoretical explanation of how the currently observed Universe was formed at the early stages. Alongside with observations theoretical approaches also show how nice inflation can be connected to the nucleosynthesis and subsequent appearance of the particle standard model. A number of inflationary scenarios are reviewed in [2] and references therein, for instance.

Even though inflation is a great model for many reasons it has problems one of which is the lack of the UV completion. To be more precise it is not UV-complete in the framework of the Einstein's General Relativity (GR) since geodesics are not past-complete. This is a general statement and it is known as the Big Bang singularity elaborated in [3-5]. One can find that alternatives to Big Bang such as "emergent" Universe or bouncing Universe [6] hit the singularity theorem by Hawking and Penrose [7] as long as we are in GR and the space-time is of the Friedmann-Lemaître-Robertson-Walker (FLRW) type.

One of the possible resolution is a modification of GR. This can be done in general in a number of ways and one may have an insight in this using the review paper [8] and references therein, for example. It is inevitable that any modification of gravity introduces higher derivatives and only special structures like Gauss-Bonnet term or Lovelock terms in general $[8,9]$ preserve the second order of the equations of motion but this is applicable only in more than 4 dimensions. On the other hand finite higher derivatives lead to ghosts due to the Ostrogradski theorem [10]. Having all orders of higher derivatives may open a way to evade the Ostrogradski statement and a successful attempt in this direction was made considering a special class of gravity modifications where higher curvature corrections are 
accompanied with non-local operators [11, 12]. Analysis in those papers shows how one can construct a ghost-free and asymptotically free modification of GR featuring a non-singular bouncing solution, and which resembles the GR itself in the IR limit. ${ }^{1}$. The further study of this model [18] has shown that the model features the expected perturbative spectrum at late times and is stable with respect to small isotropic inhomogeneous perturbations during the bounce phase and in parallel a more general model [19] (see also [20]) was proposed and considered in the Minkowski background.

Absolutely the non-local operators is what makes these models novel and the operators in question are of type of analytic functions of the covariant d'Alembertian operator, i.e. $\mathcal{F}(\square) .{ }^{2}$ We find it is quite important that the initial inspiration for introducing these operators came from string field theory (SFT) models which as the whole theory is a UV-complete non-perturbative description of strings. We refer the reader to more stringy oriented literature [23-28] for a more comprehensive overview of this aspect. A decent progress was achieved in studying non-local scalar field models derived from SFT in the cosmological context [29]-[32] as well as exploration of other aspects of this type of models including their thermodynamics [33-35]. The major question of rigorous derivation of the modified GR action involving the non-local operators of interest from the scratch, i.e. from the closed SFT, is still awaiting for its resolution but this is beyond the scope of our present study.

The present paper is aimed at extending and continuing both papers [18, 19]. In [18] a lot of technical issues were solved for a model which contains the scalar curvature squared non-local term. The main focus there is perturbations around a bouncing solution. In [19] non-local terms containing the Ricci and Riemann tensors squared were added but only analyzed around the Minkowski background. We literally want to join those works in the present paper. We confine ourselves by considering the FLRW type of the metric and positive cosmological term $\Lambda$. Having these in mind we focus on deriving full equations of motion and bringing them to a form one can use in the future study. Our main goal is to present here classical solutions and develop the perturbation technique at least in some regimes or around some backgrounds. This is crucial for claiming the model is viable or not for the purpose of describing the non-singular bounce.

The paper is organized as follows: In Section 2, we formulate the model and introduce the relevant notations. Also we write down full equations of motion relevant for the FLRW type metric. In Section 3, we demonstrate explicitly that one of the known bouncing solutions, namely the cosine hyperbolic, is the solution to our extended equations of motion as well. In Section 4, we perform an attempt to construct more solutions and develop relevant techniques. In Section 5, we are building the road to analyzing perturbation. We find out we are lucky to draw the stability statement about our exact bouncing solution from general arguments as well as sketch the derivation of the closed system of perturbation equations for the de Sitter asymptotic. In Section 6, we summarize what is done and formulate open questions for the future study.

\footnotetext{
${ }^{1}$ Similar approaches involving non-local models were used in other cosmological and gravity contexts in the literature [13-17].

${ }^{2}$ Theoretically motivated operators such as $1 / \square$ were considered, for instance, in $[21,22]$ and references therein.
} 


\section{Action and equations of motion}

We focus on the model described by the following non-local action

$$
S=\int d^{4} x \sqrt{-g}\left(\frac{M_{P}^{2}}{2} R+\frac{\lambda}{2}\left(R \mathcal{F}_{1}(\square) R+R_{\nu}^{\mu} \mathcal{F}_{2}(\square) R_{\mu}^{\nu}+C_{\mu \nu \alpha \beta} \mathcal{F}_{4}(\square) C^{\mu \nu \alpha \beta}\right)-\Lambda\right)
$$

where we limit ourselves with $O\left(R^{2}\right)$ corrections. Here the dimensionality is manifest and in the sequel all the formulae are written having 4 dimensions in mind, $M_{P}$ is the Planckian mass, $\Lambda$ is a cosmological constant and $\lambda$ is a dimensionless parameter measuring the effect of the $O\left(R^{2}\right)$ corrections. The most novel and crucial for our analysis ingredients are the functions of the covariant d'Alembertian operator $\mathcal{F}_{I}$. For simplicity to avoid extra complications we assume that these function are analytic with real coefficients $f_{I_{n}}$ in Taylor series expansion $\mathcal{F}_{I}=\sum_{n \geq 0} f_{I_{n}} \square^{n} / M_{*}^{2 n}$. The new mass scale determines the characteristic scale of the gravity modification. We assume it universal for all $\mathcal{F}_{I}$ and refer the reader to [11] for a detailed discussion of this new physics parameter. Also apart from the canonical usage of the Riemann tensor we use the Weyl tensor $C_{\alpha \nu \beta}^{\mu}$ which is coming from the Ricci decmposition

$$
C_{\nu \beta}^{\mu \alpha}=R_{\nu \beta}^{\mu \alpha}-\frac{1}{2}\left(\delta_{\nu}^{\mu} R_{\beta}^{\alpha}-\delta_{\beta}^{\mu} R_{\nu}^{\alpha}+R_{\nu}^{\mu} \delta_{\beta}^{\alpha}-R_{\beta}^{\mu} \delta_{\nu}^{\alpha}\right)+\frac{R}{6}\left(\delta_{\nu}^{\mu} \delta_{\beta}^{\alpha}-\delta_{\beta}^{\mu} \delta_{\nu}^{\alpha}\right)
$$

In this formula we use slightly unusual position of indexes which is useful in performing further computations. The reason to use the Weyl tensor is because $C_{\alpha \nu \beta}^{\mu}=0$ on a conformally flat manifold which is the case for the FLRW metric. We are focused on the FLRW cosmologies and thus will benefit out of this. Indeed, it means that the Weyl tensor squared does not show up in the background at all and only becomes relevant in perturbations. Moreover, even in perturbations the only non-vanishing contribution is the one where both Weyl tensors are perturbed and the non-local functions $\mathcal{F}_{4}$ takes its background form.

This action appears in $[19]^{3}$ and is the most general covariant non-local action up to the square in curvature terms and analytic operator functions $\mathcal{F}_{I}{ }^{4}$ Furthermore we note that working in 4 dimensions we can assume $f_{20}=0$ because using that the Gauss-Bonnet scalar is a total derivative and combining this with the Ricci decomposition we can have only $R^{2}$ when no d'Alembertian operators are in between. In other words among the terms without d'Alembertian operator insertions only $R^{2}$ survives on the FLRW backgrounds. This does not work for non-constant terms in $\mathcal{F}_{I}$ though.

Equations of motion for action (2.1) can be derived by a straightforward variation and are as follows

$$
\begin{aligned}
& {\left[M_{P}^{2}+2 \lambda \mathcal{F}_{1}(\square) R\right] G_{\nu}^{\mu}=T_{\nu}^{\mu}-\Lambda \delta_{\nu}^{\mu}-\frac{\lambda}{2} R \mathcal{F}_{1}(\square) R \delta_{\nu}^{\mu}+2 \lambda\left(\nabla^{\mu} \partial_{\nu}-\delta_{\nu}^{\mu} \square\right) \mathcal{F}_{1}(\square) R-} \\
- & 2 \lambda R_{\beta}^{\mu} \mathcal{F}_{2}(\square) R_{\nu}^{\beta}+\frac{\lambda}{2} \delta_{\nu}^{\mu} R_{\beta}^{\alpha} \mathcal{F}_{2}(\square) R_{\alpha}^{\beta}+ \\
+ & 2 \lambda\left(\nabla_{\rho} \nabla_{\nu} \mathcal{F}_{2}(\square) R^{\mu \rho}-\frac{1}{2} \square \mathcal{F}_{2}(\square) R_{\nu}^{\mu}-\frac{1}{2} \delta_{\nu}^{\mu} \nabla_{\sigma} \nabla_{\rho} \mathcal{F}_{2}(\square) R^{\sigma \rho}\right)+ \\
+ & \lambda \mathcal{K}_{1}^{\mu}{ }_{\nu}^{\mu}-\frac{\lambda}{2} \delta_{\nu}^{\mu}\left(\mathcal{K}_{1 \sigma}^{\sigma}+\overline{\mathcal{K}}_{1}\right)+\lambda \mathcal{K}_{2}^{\mu}-\frac{\lambda}{2} \delta_{\nu}^{\mu}\left(\mathcal{K}_{2 \sigma}^{\sigma}+\overline{\mathcal{K}}_{2}\right)+2 \lambda \Delta_{\nu}^{\mu}+2 \lambda \mathcal{C}_{\nu}^{\mu}
\end{aligned}
$$

\footnotetext{
${ }^{3}$ It is eq. (27) there. We have however less terms because possible contractions of the covariant derivatives with the Ricci tensor can be eliminated thanks to the Bianchi identity or converted to the higher order in curvature terms using the commutation relations for the covariant derivatives.

${ }^{4}$ Also questions of a ghost-free gravity modification are addressed in [36] using different action still using non-local operators constructed out of the d'Alembertian operator.
} 
where we have defined:

$$
\begin{aligned}
& \mathcal{K}_{1}^{\mu}=\sum_{n=1}^{\infty} f_{1 n} \sum_{l=0}^{n-1} \partial^{\mu} R^{(l)} \partial_{\nu} R^{(n-l-1)}, \overline{\mathcal{K}}_{1}=\sum_{n=1}^{\infty} f_{1 n} \sum_{l=0}^{n-1} R^{(l)} R^{(n-l)}, \\
& \mathcal{K}_{2}{ }_{\nu}^{\mu}=\sum_{n=1}^{\infty} f_{2 n} \sum_{l=0}^{n-1} \nabla^{\mu} R^{(l)}{ }_{\beta}^{\alpha} \nabla_{\nu} R^{(n-l-1)}{ }_{\alpha}^{\beta}, \overline{\mathcal{K}}_{2}=\sum_{n=1}^{\infty} f_{2 n} \sum_{l=0}^{n-1} R_{\beta}^{(l)}{ }_{\beta}^{\alpha} R^{(n-l)}{ }_{\alpha}^{\beta}, \\
& \Delta_{\nu}^{\mu}=\sum_{n=1}^{\infty} f_{2 n} \sum_{l=0}^{n-1} \nabla_{\beta}\left[R_{\gamma}^{(l)}{ }_{\gamma}^{\beta} \nabla^{\mu} R_{\nu}^{(n-l-1)^{\gamma}}-\nabla^{\mu} R_{\gamma}^{(l)} R_{\nu}^{(n-l-1)}{ }_{\nu}^{\gamma}\right]
\end{aligned}
$$

and $\mathcal{C}_{\nu}^{\mu}$ is the part coming from the variation of the Weyl tensor squared piece. As far as we mostly concerned about FLRW cosmologies we simply neglect this contribution in the background as it is zero. We shall return to it later during the consideration of perturbations. Here $G_{\nu}^{\mu}$ is the Einstein tensor, $R^{(n)}=\square^{n} R, R_{\beta}^{(n)^{\alpha}}=\square^{n} R_{\beta}^{\alpha}$, and $T_{\nu}^{\mu}$ is the matter stress tensor if one is present in the system.

One would benefit considering first the trace equation

$$
\begin{aligned}
& -M_{P}^{2} R=T-4 \Lambda-6 \lambda \square \mathcal{F}_{1}(\square) R-\lambda\left(\mathcal{K}_{1}+2 \overline{\mathcal{K}}_{1}\right)- \\
& -\lambda \square \mathcal{F}_{2}(\square) R-2 \lambda \nabla_{\rho} \nabla_{\mu} \mathcal{F}_{2}(\square) R^{\mu \rho}-\lambda\left(\mathcal{K}_{2}+2 \overline{\mathcal{K}}_{2}\right)+2 \lambda \Delta+2 \lambda \mathcal{C}
\end{aligned}
$$

where quantities without indices denote the trace and significant simplifications are obvious.

\section{Exact solutions based on the recursion relations}

\subsection{Recursion relations}

In what follows in this Section we use quite intensively ideas and the knowledge accumulated in [18] (see also [12]) where this model without $\mathcal{F}_{2}$ and $\mathcal{F}_{4}$ pieces was considered and solutions (and their construction) were discussed. We therefore omit extensive citing of these papers and do refer the reader to those manuscripts for a detailed analysis of a model which is $\mathcal{F}_{2}=\mathcal{F}_{4}=0$ in our notations.

Clearly those equations have as solutions Minkowski (if $\Lambda=0$ ) and de Sitter (if $\Lambda=$ const $>0$ ) backgrounds. The question is however whether we can find other solutions especially focusing on the possible bouncing backgrounds. Equations are definitely highly non-trivial but a decent progress was achieved in [11] by employing the ansatz

$$
\square R=r_{1} R+r_{2}
$$

in the absence of $\mathcal{F}_{2}$ and $\mathcal{F}_{4}$. This makes the system much simpler creating the recursive relation for $\square^{n}$ and one can try to find exact solutions. Known exact solutions (apart from $M_{4}$ and $d S_{4}$ ) include

$$
a(t)=a_{0} \cosh (\sigma t) \text { found in }[11]
$$

and

$$
a(t)=a_{0} \exp \left(\frac{\sigma}{2} t^{2}\right) \text { found in [37]. }
$$

where $a(t)$ is the scale factor of the FLRW metric $g_{\mu \nu}=\operatorname{diag}\left(-1, a(t)^{2}, a(t)^{2}, a(t)^{2}\right)$. 
For the Ricci tensor term we suggest the following relation as a quite general ansatz

$$
\square R_{\mu \nu}^{(n)}=\sum_{m=0}^{n}\left(r_{1 m} R_{\mu \nu}^{(m)}+r_{2 m} g_{\mu \nu} R^{(m)}\right)+r g_{\mu \nu}
$$

where $r_{1,2 m}$ and $r$ are constants. Surely we require $n$ to be finite. This would manifest the recursion relation when acting by extra $\square$-s and give us a chance to have this as an exact solution to the equations (2.2). Needless to say that smaller $n$ makes the succeeding analysis simpler.

The explanation why finding of such a recursive relation is almost enough is based on the following arguments. One should start with the trace equation (2.3). If there is no matter or only radiation (which is conformal and traceless) is present than the trace equation contains only the gravitational sector of the model. It can be solved simply by imposing null conditions on coefficients in front of different powers of the curvature. These are some conditions on the model parameters but clearly the restrictions are very weak as far as $n$ is finite. Further we must check one of the component equations (we shall do for (00) component) in (2.2) whether it is satisfied or not. But thanks to the Bianchi identity constraint any discrepancy we can meet would be of the form of the radiation energy density, i.e. $\sim a(t)^{-4}$. Hence the only question would be how much radiation must we inject in the system and would it have good or ghost sign of the energy density.

Of course the proposed above ansatz is of extreme generality and in order to be more specific we take its following simplified version into the consideration

$$
\square^{n} \tilde{G}_{\nu}^{\mu}=\alpha_{n} \square \tilde{G}_{\nu}^{\mu}+\beta_{n} \tilde{G}_{\nu}^{\mu}, n \geq 2, \text { where } \tilde{G}_{\nu}^{\mu}=R_{\nu}^{\mu}-\frac{1}{4} \delta_{\nu}^{\mu} R
$$

The recursion relation for $n=2$ can be easily solved ${ }^{5}$ to give

$$
\alpha_{n}=c_{+} s_{+}^{n}+c_{-} s_{-}^{n}, s_{ \pm}=\frac{\alpha_{2} \pm \sqrt{\alpha_{2}^{2}+4 \beta_{2}}}{2}, \beta_{n}=\alpha_{n-1} \beta_{2}
$$

and $c_{ \pm}$are to be found examining explicit values of $\alpha_{3}, \beta_{3}$. However there is a limiting value $n=4$. $n \leq 4$ gives a hypothetical chance to solve the recursion relation while greater $n$ will result in general in algebraic equations of a degree higher than 4 .

\subsection{Exact analytic bounce}

This choice of the simplified ansatz is motivated by the fact that for solution (3.2) we have after some simplifications

$$
\square^{2} \tilde{G}_{\nu}^{\mu}=14 \sigma^{2} \square \tilde{G}_{\nu}^{\mu}-40 \sigma^{4} \tilde{G}_{\nu}^{\mu}
$$

Below we mainly study the particular solution (3.2) meaning that we have specific $s_{ \pm}, c_{ \pm}$. One can, surely, track all the steps considering (3.5) in general without specifying particular values for $\alpha_{2}$ and $\beta_{2}$. This is interesting in case more solutions satisfying this ansatz can be found. Going general one would come to a bit more complicated expressions than we will. We do not do this at the moment for the sake of clarity. Moreover (3.5) is already the simplified version of (3.4) which is worth to analyse if a really general scenario is of interest. We thus focus mostly on analysing found solution (3.2).

\footnotetext{
${ }^{5}$ We thank at this point Dan Thompson for illuminating this issue.
} 
On solution (3.2) we find

$$
\alpha_{n}=\frac{1}{6 \sigma^{2}}\left(s_{1}^{n}-s_{2}^{n}\right), \beta_{n}=-\frac{40 \sigma^{2}}{6}\left(s_{1}^{n-1}-s_{2}^{n-1}\right), s_{1}=10 \sigma^{2}, s_{2}=4 \sigma^{2} .
$$

Note that this relations can be used for $n=1$ and $n=0$ as they generate correct values $\alpha_{1}=1, \beta_{1}=0$ and $\alpha_{0}=0, \beta_{0}=1$ respectively which are reasonable. Even though $f_{2_{0}}=0$ as explained in the previous Section we shall use this property formally to simplify the evaluation of the sums in the equations of motion. Apart from this the first ansatz (3.1) is also satisfied with $r_{1}=2 \sigma^{2}, r_{2}=-24 \sigma^{4}$. We thus have

$$
\square^{n} R=r_{1}^{n}\left(R+r_{2} / r_{1}\right), \square^{n} \tilde{G}_{\nu}^{\mu}=\frac{s_{1}^{n}}{6 \sigma^{2}}\left(\square \tilde{G}_{\nu}^{\mu}-s_{2} \tilde{G}_{\nu}^{\mu}\right)-\frac{s_{2}^{n}}{6 \sigma^{2}}\left(\square \tilde{G}_{\nu}^{\mu}-s_{1} \tilde{G}_{\nu}^{\mu}\right)
$$

These relations yield

$$
\begin{aligned}
& \mathcal{K}_{1_{\nu}^{\mu}}^{\mu}=\mathcal{F}_{1}^{(1)}\left(r_{1}\right) \partial^{\mu} R \partial_{\nu} R, \\
& \overline{\mathcal{K}}_{1}=r_{1} \mathcal{F}_{1}^{(1)}\left(r_{1}\right) R^{2}+2 r_{2} \mathcal{F}_{1}^{(1)}\left(r_{1}\right) R-\left(\mathcal{F}_{1}\left(r_{1}\right)-f_{1_{0}}\right) R \frac{r_{2}}{r_{1}}+ \\
& +\frac{r_{2}^{2}}{r_{1}} \mathcal{F}_{1}^{(1)}\left(r_{1}\right)-\left(\mathcal{F}_{1}\left(r_{1}\right)-f_{10}\right)\left(r_{2} / r_{1}\right)^{2} \\
& \mathcal{K}_{2}^{\mu}{ }_{\nu}^{\mu}=\mathcal{F}_{2}^{(1)}\left(s_{1}\right) \nabla^{\mu} S_{1 \beta}^{\alpha} \nabla_{\nu} S_{1}^{\beta}{ }_{\alpha}+\mathcal{F}_{2}^{(1)}\left(s_{2}\right) \nabla^{\mu} S_{2}{ }_{\beta}^{\alpha} \nabla_{\nu} S_{2}{ }_{\alpha}^{\beta}- \\
& -\frac{\mathcal{F}_{2}\left(s_{1}\right)-\mathcal{F}_{2}\left(s_{2}\right)}{s_{1}-s_{2}}\left(\nabla^{\mu} S_{1}^{\alpha}{ }_{\beta}^{\alpha} \nabla_{\nu} S_{2}^{\beta}{ }_{\alpha}+\nabla^{\mu} S_{2}^{\alpha} \nabla_{\nu} S_{1}^{\beta}\right)+\frac{1}{4} \mathcal{F}_{2}^{(1)}\left(r_{1}\right) \partial^{\mu} R \partial_{\nu} R, \\
& \overline{\mathcal{K}}_{2}=s_{1} \mathcal{F}_{2}^{(1)}\left(s_{1}\right) S_{1}{ }_{\beta}^{\alpha} S_{1}{ }_{\alpha}^{\beta}+s_{2} \mathcal{F}_{2}^{(1)}\left(s_{2}\right) S_{2}{ }_{\beta}^{\alpha} S_{2}{ }_{\alpha}^{\beta}-\frac{\mathcal{F}_{2}\left(s_{1}\right)-\mathcal{F}_{2}\left(s_{2}\right)}{s_{1}-s_{2}}\left(s_{1}+s_{2}\right) S_{1}{ }_{\beta}^{\alpha} S_{2}{ }_{\alpha}^{\beta}+ \\
& +\frac{1}{4}\left(r_{1} \mathcal{F}_{2}^{(1)}\left(r_{1}\right) R^{2}+2 r_{2} \mathcal{F}_{2}^{(1)}\left(r_{1}\right) R-\mathcal{F}_{2}\left(r_{1}\right) R \frac{r_{2}}{r_{1}}+\frac{r_{2}^{2}}{r_{1}} \mathcal{F}_{2}^{(1)}\left(r_{1}\right)-\mathcal{F}_{2}\left(r_{1}\right)\left(r_{2} / r_{1}\right)^{2}\right), \\
& \Delta_{\nu}^{\mu}=\nabla_{\beta}\left[\mathcal{F}_{2}^{(1)}\left(s_{1}\right)\left(S_{1 \gamma}^{\beta} \nabla^{\mu} S_{1 \nu}^{\gamma}-\nabla^{\mu} S_{1 \gamma}^{\beta} S_{1 \nu}^{\gamma}\right)+\mathcal{F}_{2}^{(1)}\left(s_{2}\right)\left(S_{2 \gamma}^{\beta} \nabla^{\mu} S_{2 \nu}^{\gamma}-\nabla^{\mu} S_{2 \gamma}^{\beta} S_{2 \nu}^{\gamma}\right)-\right. \\
& \left.-\frac{\mathcal{F}_{2}\left(s_{1}\right)-\mathcal{F}_{2}\left(s_{2}\right)}{s_{1}-s_{2}}\left(S_{1 \gamma}^{\beta} \nabla^{\mu} S_{2 \nu}^{\gamma}-\nabla^{\mu} S_{1 \gamma}^{\beta} S_{2 \nu}^{\gamma}+S_{2 \gamma}^{\beta} \nabla^{\mu} S_{1 \nu}^{\gamma}-\nabla^{\mu} S_{2 \gamma}^{\beta} S_{1 \nu}^{\gamma}\right)\right] \text {, } \\
& \mathcal{F}_{1}(\square) R=\mathcal{F}_{1}\left(r_{1}\right) R+\left(\mathcal{F}_{1}\left(r_{1}\right)-f_{0}\right) \frac{r_{2}}{r_{1}}, \mathcal{F}_{2}(\square) R_{\nu}^{\mu}=\mathcal{F}_{2}\left(s_{1}\right) S_{1}^{\mu}-\mathcal{F}_{2}\left(s_{2}\right) S_{2}{ }_{\nu}^{\mu}+\frac{1}{4} \delta_{\nu}^{\mu} \mathcal{F}_{2}(\square) R
\end{aligned}
$$

where we have defined

$$
S_{1}^{\mu}=\frac{\square \tilde{G}_{\nu}^{\mu}-s_{2} \tilde{G}_{\nu}^{\mu}}{6 \sigma^{2}}, S_{2}^{\mu}=\frac{\square \tilde{G}_{\nu}^{\mu}-s_{1} \tilde{G}_{\nu}^{\mu}}{6 \sigma^{2}} .
$$

The slightly counter-intuitive remixed position of subscripts 1,2 is correct here. $\mathcal{F}_{1,2}^{(1)}$ is the derivative with respect to the argument. In order to cancel in the trace equation structures containing $\square \tilde{G}_{\nu}^{\mu}$ and $\left(\square \tilde{G}_{\nu}^{\mu}\right)^{2}$ we have to impose the following conditions

$$
\mathcal{F}_{2}^{(1)}\left(s_{1}\right)=\mathcal{F}_{2}^{(1)}\left(s_{2}\right)=\mathcal{F}_{2}\left(s_{1}\right)-\mathcal{F}_{2}\left(s_{2}\right)=0
$$

and to cancel structures containing $\left(\partial_{\mu} R\right)^{2}$ and $R^{2}$

$$
\mathcal{F}_{1}^{(1)}\left(r_{1}\right)+\frac{1}{4} \mathcal{F}_{2}^{(1)}\left(r_{1}\right)=0
$$


All other equations are also simplified on imposing the above conditions. In particular we have

$$
\mathcal{F}_{2}(\square) R_{\nu}^{\mu}=\mathcal{F}_{2}\left(s_{1}\right)\left(G_{\nu}^{\mu}+\frac{1}{4} \delta_{\nu}^{\mu} R\right)+\frac{1}{4} \delta_{\nu}^{\mu} \mathcal{F}_{2}\left(r_{1}\right)\left(R+r_{2} / r_{1}\right)
$$

or equivalently

$$
\mathcal{F}_{2}(\square) \tilde{G}_{\nu}^{\mu}=\mathcal{F}_{2}\left(s_{1}\right) \tilde{G}_{\nu}^{\mu} .
$$

Careful substitution in the trace of the Einstein equations (2.3) reads

$$
\begin{aligned}
& -M_{P}^{2} R=T-4 \Lambda-6 \lambda\left(\mathcal{F}_{1}\left(r_{1}\right)+\frac{1}{4} \mathcal{F}_{2}\left(r_{1}\right)\right)\left(r_{1} R+r_{2}\right)-\frac{\lambda}{2} \mathcal{F}_{2}\left(s_{1}\right)\left(r_{1} R+r_{2}\right)+ \\
& +2 \lambda\left(\mathcal{F}_{1}\left(r_{1}\right)+\frac{1}{4} \mathcal{F}_{2}\left(r_{1}\right)-f_{10}\right) \frac{r_{2}}{r_{1}}\left(R+r_{2} / r_{1}\right)
\end{aligned}
$$

and we solve it (assuming $T=0$ ) by imposing

$$
\begin{aligned}
\frac{M_{P}^{2}}{r_{1}}-\frac{\lambda}{2} \mathcal{F}_{2}\left(s_{1}\right)-6 \lambda\left(\mathcal{F}_{1}\left(r_{1}\right)+\frac{1}{4} \mathcal{F}_{2}\left(r_{1}\right)\right)+2 \lambda\left(\mathcal{F}_{1}\left(r_{1}\right)+\frac{1}{4} \mathcal{F}_{2}\left(r_{1}\right)-f_{10}\right) \frac{r_{2}}{r_{1}^{2}} & =0 \\
-\frac{M_{P}^{2}}{4} \frac{r_{2}}{r_{1}} & =\Lambda
\end{aligned}
$$

These relations in terms of $\sigma$ are as follows

$$
\begin{aligned}
\frac{M_{P}^{2}}{2 \sigma^{2}}-\frac{\lambda}{2} \mathcal{F}_{2}\left(4 \sigma^{2}\right)-18 \lambda \mathcal{F}_{1}\left(2 \sigma^{2}\right)-\frac{9}{2} \lambda \mathcal{F}_{2}\left(2 \sigma^{2}\right)+12 \lambda f_{10} & =0 \\
3 M_{P}^{2} \sigma^{2} & =\Lambda
\end{aligned}
$$

Even though some tuning is required for functions $\mathcal{F}_{1,2}$ the solution to the above conditions is really ambiguous.

Substituting the obtained results in (00) component of (2.2) and performing a number of manipulations we find that only terms proportional to a constant, $1 / \cosh (\sigma t)^{2}$ and $1 / \cosh (\sigma t)^{4}$ are present and moreover upon applying the above conditions (3.12) one is left with the following combination

$$
\rho_{r}=-\frac{54 \lambda \sigma^{4}}{\cosh (\sigma t)^{4}}\left(\mathcal{F}_{1}\left(r_{1}\right)+\frac{1}{4} \mathcal{F}_{2}\left(r_{1}\right)+\frac{1}{12} \mathcal{F}_{2}\left(s_{2}\right)\right)
$$

where we clearly see that we are really left with terms resembling the radiation (i.e. $\sim a(t)^{-4}$ ). What is remarkable here that there are two possible resolutions to have the equations solved completely.

First way is to avoid extra radiation requiring

$$
\mathcal{F}_{1}\left(r_{1}\right)+\frac{1}{4} \mathcal{F}_{2}\left(r_{1}\right)+\frac{1}{12} \mathcal{F}_{2}\left(s_{2}\right)=0
$$

This means that extra parameter adjustment is important which however does not seem to be unnatural. Equations do not trivialize in this case and we can analyse such a configuration consistently. Note, it is not the case if $\mathcal{F}_{2}=0$ (see [18] for details).

Second way is to admit some amount of radiation with a weaker requirement

$$
\mathcal{F}_{1}\left(r_{1}\right)+\frac{1}{4} \mathcal{F}_{2}\left(r_{1}\right)+\frac{1}{12} \mathcal{F}_{2}\left(s_{2}\right)<0
$$


so that this radiation has the positive energy and is not a ghost.

We therefore see that solution (3.2) equipped with conditions $(3.8,3.9)$ features a number of very nice properties: (i) it has non-singular bounce, (ii) it comes at large time to the de Sitter phase rather than eternal superinflation, (iii) it requires just few fine-tunings in the system which still leave us with an enormous freedom and (iv) it does not have to be supported by extra sources if we impose an extra condition (3.14) (to avoid this extra tuning we would assume (3.15) to avoid ghosts in the system).

\section{More analytic cosmological solutions?}

In this Section we provide several solutions which unfortunately in most cases do not contribute to the question of the non-singular bounce but are nevertheless interesting for the future developments in this type of theories.

\subsection{Closely related solutions}

Apart from solution (3.2) analyzed in the previous Section one can find after simple analysis that the following are also analytic solutions:

$$
\begin{aligned}
& a(t)=a_{0} \sinh (\sigma t) \\
& a(t)=a_{0} \cos (\sigma t)
\end{aligned}
$$

They can be quite simply obtained using the already known one and unfortunately none of them contributes to our main subject of a non-singular bounce. Indeed

- Solution (4.1a) is clearly not a bouncing solution and we mention it here just for the sake of completeness.

- Solution (4.1b) is just our already explored solution (3.2) with an imaginary parameter $\sigma$. It has periodic zeros for the scale factor. It can be used as a test-bed for discussing possible stages of the Universe evolution if they obey $\cos (\sigma t)$ behaviour.

Obviously there should be other solutions in the system. At first let us mention that (3.3) does not generate a recursive relation for the Ricci tensor terms. One could think also that there is a possibility to solve equation (3.10) just as a differential equation without adjusting the coefficients but this turns out to be incompatible with the ansatzes. Indeed, equation (3.10) was obtained using (3.1) and (3.5) while a solution to it does not satisfy these ansatzes identically. Even just finding a solution other then presented in the previous Section to the ansatzes conditions is not an easy task as it comes to solving at least the third order non-linear differential equation. As of now it is an open question how to construct more solutions in this model using some ansatz (see [38] for various proposal regarding solving the ansatz condition).

So on one hand we absolutely understand that taming all the non-localities by virtue of a recursion relation is the good technical point of the all considered above solutions. This also must help in studying perturbations around those particular backgrounds. On the other hand however this significantly reduces the possible solutions. We thus want to go beyond the recursion relations and to find a more general approach to the problem of solving equations of motion. 


\subsection{Model reformulation using $\tilde{G}_{\nu}^{\mu}$}

The first useful technical step is a passage from the Ricci tensor to the traceless analog of the Einstein tensor $\tilde{G}_{\nu}^{\mu}$. We mention in this regard that appearance of a combination $\mathcal{F}_{1}+\frac{1}{4} \mathcal{F}_{2}$ is not spontaneous because we can rewrite the initial action (2.1) in terms of $\tilde{G}_{\nu}^{\mu}$ as follows

$$
S=\int d^{4} x \sqrt{-g}\left(\frac{M_{P}^{2}}{2} R+\frac{\lambda}{2}\left(R \tilde{\mathcal{F}}_{1}(\square) R+\tilde{G}_{\nu}^{\mu} \mathcal{F}_{2}(\square) \tilde{G}_{\mu}^{\nu}+C_{\mu \nu \alpha \beta} \mathcal{F}_{4}(\square) C^{\mu \nu \alpha \beta}\right)-\Lambda\right)
$$

where we have used that $\tilde{G}_{\nu}^{\mu}$ is traceless and have defined $\mathcal{F}_{1}(\square)+\frac{1}{4} \mathcal{F}_{2}(\square)=\tilde{\mathcal{F}}_{1}(\square)$. Equations of motion for action (4.2) can be derived by substituting $R_{\nu}^{\mu}=\tilde{G}_{\nu}^{\mu}+\frac{1}{4} \delta_{\nu}^{\mu} R$ in (2.2)

$$
\begin{aligned}
& M_{P}^{2} G_{\nu}^{\mu}=T_{\nu}^{\mu}-\Lambda \delta_{\nu}^{\mu}-2 \lambda \tilde{G}_{\nu}^{\mu} \tilde{\mathcal{F}}_{1}(\square) R+2 \lambda\left(\nabla^{\mu} \partial_{\nu}-\delta_{\nu}^{\mu} \square\right) \tilde{\mathcal{F}}_{1}(\square) R-\frac{1}{2} \lambda R \mathcal{F}_{2}(\square) \tilde{G}_{\nu}^{\mu}- \\
& -2 \lambda \tilde{G}_{\beta}^{\mu} \mathcal{F}_{2}(\square) \tilde{G}_{\nu}^{\beta}+\frac{\lambda}{2} \delta_{\nu}^{\mu} \tilde{G}_{\beta}^{\alpha} \mathcal{F}_{2}(\square) \tilde{G}_{\alpha}^{\beta}+ \\
& +2 \lambda\left(\nabla_{\rho} \nabla_{\nu} \mathcal{F}_{2}(\square) \tilde{G}^{\mu \rho}-\frac{1}{2} \square \mathcal{F}_{2}(\square) \tilde{G}_{\nu}^{\mu}-\frac{1}{2} \delta_{\nu}^{\mu} \nabla_{\sigma} \nabla_{\rho} \mathcal{F}_{2}(\square) \tilde{G}^{\sigma \rho}\right)+ \\
& +\lambda \mathcal{L}_{1}^{\mu}-\frac{\lambda}{2} \delta_{\nu}^{\mu}\left(\mathcal{L}_{1 \sigma}^{\sigma}+\overline{\mathcal{L}}_{1}\right)+\lambda \mathcal{L}_{2}^{\mu}-\frac{\lambda}{2} \delta_{\nu}^{\mu}\left(\mathcal{L}_{2 \sigma}^{\sigma}+\overline{\mathcal{L}}_{2}\right)+2 \lambda \tilde{\Delta}_{\nu}^{\mu}+2 \lambda \mathcal{C}_{\nu}^{\mu}
\end{aligned}
$$

where we have defined:

$$
\begin{gathered}
\mathcal{L}_{1}{ }_{\nu}^{\mu}=\sum_{n=1}^{\infty} \tilde{f}_{1 n} \sum_{l=0}^{n-1} \partial^{\mu} R^{(l)} \partial_{\nu} R^{(n-l-1)}, \overline{\mathcal{L}}_{1}=\sum_{n=1}^{\infty} \tilde{f}_{1 n} \sum_{l=0}^{n-1} R^{(l)} R^{(n-l)}, \\
\mathcal{L}_{2}{ }_{\nu}^{\mu}=\sum_{n=1}^{\infty} f_{2 n} \sum_{l=0}^{n-1} \nabla^{\mu} \tilde{G}_{\beta}^{(l) \alpha} \nabla_{\nu} \tilde{G}_{\alpha}^{(n-l-1) \beta}, \overline{\mathcal{L}}_{2}=\sum_{n=1}^{\infty} f_{2 n} \sum_{l=0}^{n-1} \tilde{G}_{\beta}^{(l) \alpha} \tilde{G}_{\alpha}^{(n-l) \beta}, \\
\tilde{\Delta}_{\nu}^{\mu}=\sum_{n=1}^{\infty} f_{2 n} \sum_{l=0}^{n-1} \nabla_{\beta}\left[\tilde{G}_{\gamma}^{(l) \beta} \nabla^{\mu} \tilde{G}_{\nu}^{(n-l-1) \gamma}-\nabla^{\mu} \tilde{G}_{\gamma}^{(l) \beta} \tilde{G}_{\nu}^{(n-l-1) \gamma}\right]
\end{gathered}
$$

and $\tilde{f}_{1 n}$ are coefficients of the Taylor expansion of function $\tilde{\mathcal{F}}_{1}$. The Weyl tensor related part may have an impact now since we are going to consider perturbations. One can find the relevant part of it is

$$
\mathcal{C}_{\nu}^{\mu}=\left(R_{\alpha \beta}+2 \nabla_{\alpha} \nabla_{\beta}\right) \mathcal{F}_{4}(\square) C_{\nu}{ }^{\alpha \beta \mu} .
$$

Saying relevant we mean only the piece which is obtained by the variation of one of the Weyl tensor factors in the action. Then we are left with only one Weyl tensor as it is obvious from the latter formula and further perturbation of this remaining Wel tensor factor may produce a non-zero contribution to the perturbation equations.

The trace equation becomes

$$
\begin{aligned}
& -M_{P}^{2} R=T-4 \Lambda-6 \lambda \square \tilde{\mathcal{F}}_{1}(\square) R-\lambda\left(\mathcal{L}_{1}+2 \overline{\mathcal{L}}_{1}\right)- \\
& -2 \lambda \nabla_{\rho} \nabla_{\mu} \mathcal{F}_{2}(\square) \tilde{G}^{\mu \rho}-\lambda\left(\mathcal{L}_{2}+2 \overline{\mathcal{L}}_{2}\right)+2 \lambda \tilde{\Delta}
\end{aligned}
$$

and the Weyl tensor related term $\mathcal{C}$ turns out to be zero thanks to the full tracelessness of the Weyl tensor.

This form of action and equations of motion also turns out to be beneficial for studying perturbations as we will see in the next Section. 


\subsection{Avoiding recursion relations}

Surely we are still sticking to the FLRW type of the metric but keep up to some extent the scale factor general. This means that we cannot say anything specific about functions $R^{(n)}$ which appear after the box operator acts on the scalar curvature $n$ times. Fortunately, we now can have a tiny progress with the second rank tensor $\tilde{G}_{\nu}^{\mu}$. It is traceless and action of the box operator does not break this property because box commutes with the metric. It means that $\tilde{G}_{\mu}^{(n) \mu}=0$ for any $n$ and thus we can introduce

$$
\square^{n} \tilde{G}_{\nu}^{\mu}=b_{n}(t) \zeta_{\nu}^{\mu} \text { where } \zeta_{\nu}^{\mu}=\operatorname{diag}(3,-1,-1,-1)
$$

where one can compute that

$$
b_{n+1}(t)=\left(\square+8 H^{2}\right) b_{n}(t) \text { and } b_{0}(t)=\dot{H} / 2 .
$$

Here as usual $H=\dot{a} / a$ is the Hubble function and dot is the derivative with respect to the cosmic time $t$.

This allows us to simplify all the tensor structures in the trace equation (4.4). As was explained in the previous Section this is the cornerstone equation since a solution to it is almost automatically a solution to all the Einstein equations modulo perhaps some radiation (assuming no other matter is present in the system). Careful substitution gives

$$
\begin{aligned}
\mathcal{L}_{2} & =12 \sum_{n=1}^{\infty} f_{2 n} \sum_{l=0}^{n-1}\left(\partial^{\mu} b_{l}(t) \partial_{\mu} b_{n-l-1}(t)-8 H^{2} b_{l}(t) b_{n-l-1}(t)\right), \\
\overline{\mathcal{L}}_{2} & =12 \sum_{n=1}^{\infty} f_{2 n} \sum_{l=0}^{n-1} b_{l}(t) b_{n-l}(t), \\
\tilde{\Delta} & \left.=-48 \sum_{n=1}^{\infty} f_{2 n} \sum_{l=0}^{n-1}\left(H \partial_{t}\left(b_{l}(t) b_{n-l-1}(t)\right)+\left(3 H^{2}+\dot{H}\right) b_{l}(t) b_{n-l-1}(t)\right)\right), \\
\nabla_{\rho} \nabla_{\mu} \mathcal{F}_{2}(\square) \tilde{G}^{\mu \rho} & =3 \square b(t)-12 H(\dot{b}(t)+H b(t)) \text { where } b(t)=\sum_{n=1}^{\infty}\left(f_{2 n} b_{n}\right) .
\end{aligned}
$$

It is manifest now that we have reduced the problem of solving the trace equation to the problem of many scalar functions and moreover all of them are connected by means of the covariant box operator. To have the complete picture we note down

$$
R=12 H^{2}+6 \dot{H} \text {. }
$$

Possible recursion relations may be of course substituted here. Technically presence of a recursion relation for $R^{(n)}$ or $b_{n}$ or both means that the corresponding infinite series recurses after a certain $n$. The following configurations may appear than in this model:

1. If both $R^{(n)}$ and $b_{n}$ obey some recursion conditions for a given $a(t)$ then this in turn means that there is a finite set of linearly independent functions and in order to satisfy the trace Einstein equation we must equate to zero coefficients in front of each such function. For a finite $n$ this produces finite number of conditions on $f_{1,2_{k}}$. This is exactly what is implemented in the previous Section for the solution (3.2). 
2. If only, say, $R^{(n)}$ obeys a recursion relation while $b_{n}$ not then this means that in general infinite fine tuning is required. Indeed, absence of recursion for $b_{n}$ is equivalent to the fact that infinitely many linear independent functions will be generated all accompanied by $f_{2 n}$ coefficients. Here we see two ways to satisfy the trace Einstein equation. Either all the coefficients $f_{2 n}$ must be trivial eliminating the corresponding action modification at all or we must fine tune all of them such that the infinite series of functions sums up to a constant. The latter possibility may be accomplished in the spirit of the so called "addition theorem" for the associate Legendre functions when some certain infinite series sums up to the unit (see for instance formulae (8.794) and (8.814) in [39]). For this to happen it is vital to have infinite number of non-zero coefficients $f_{2 n}$. This is reasonable in our set-up but on the other hand all of them must be fine tuned. This is not definitely unacceptable, however. When, for example, we speak about a function like $\mathcal{F}_{2}=e^{\beta \square}$ we say it has one free parameter $\beta$ rather than infinitely many. The point here is that these operator functions come normally from the consideration of the corresponding string models as mentioned in the Introduction and their nature is therefore originates from some other principles and not from our cosmological model. Thus in the case some solution needs an infinite number of coefficients to be fixed we should only worry whether the resulting operator function is acceptable from the point of view of the string model it comes from. Example with such properties is solution (3.3).

3. If neither $R^{(n)}$ nor $b_{n}$ appear to obey a recursion all the coefficients $f_{1,2_{n}}$ are in the play. Again they can be either trivialized or fine tuned. Here, however another type of fine tuning may arise when instead of fixing all the coefficients we do fix only some relations in between of $f_{1}$-s and $f_{2}$-s. This is still an infinite fine tuning from the point of view of individual coefficients but from the point of view of the operator functions $\mathcal{F}$ this may be just one simple relation. To be more precise, provided cancellation of all terms happens if $f_{1_{n}} / f_{2_{n}}=$ const which does not depend on $n$ then it is equivalent to have $\tilde{\mathcal{F}}_{1} \sim \mathcal{F}_{2}$.

\subsection{Other solutions}

\section{Solutions of the first type.}

Solution belonging to the first type when both the scalar curvature and the Ricci tensor obey recursion relations, apart from (3.2), is

$$
a(t)=a_{0} t
$$

One can find that on this solution

$$
R=6 / t^{2}, \square R=0, b_{0}=-\frac{1}{2 t^{2}}, b_{1}=-\frac{4}{t^{2}}, b_{2}=0
$$

and it is easy to show that equations of motion can be satisfied. It is not a bouncing solution but may be interesting in analyzing the regime when the scale factor grows linearly.

\section{Configurations of the second type.}

Known configurations of the second type are as follows

$$
a(t)=a_{0} \sqrt{t}
$$


For this scale factor we have

$$
R=0, b_{0}=-\frac{1}{4 t^{2}}, b_{1}=\frac{1}{4 t^{2}}, b_{2}=-\frac{3}{t^{6}}, \ldots
$$

and sequence of $b$-s does not stop.

- Equations become a bit more transparent if we pass to $a(t)$ as the variable. We can do this in each region where we have one to one correspondence $t \leftrightarrow a(t)$. We therefore can rewrite equations in the new variable and time derivatives transform to $\partial_{t}=a H(a) \partial_{a}$. The most important for us d'Alembertian operator $\square$ becomes

$$
\square=-a^{2} H^{2}\left(\partial_{a}^{2}+\left(\frac{4}{a}+\frac{H^{\prime}}{H}\right) \partial_{a}\right)
$$

where prime is the derivative with respect to $a$. Another important operator is

$$
\square+8 H^{2}=-a^{2} H^{2}\left(\partial_{a}^{2}+\left(\frac{4}{a}+\frac{H^{\prime}}{H}\right) \partial_{a}-\frac{8}{a^{2}}\right)
$$

since it creates functions $b_{n}$.

To have a connection with the previously obtained solution note that

$$
H=\sigma \sqrt{1-\frac{a_{0}^{2}}{a^{2}}}
$$

gives $a(t)=a_{0} \cosh (\sigma t)$.

Than one can guess some interesting configurations and one of them is

$$
H=\sigma \sqrt{1-\frac{a_{0}^{4}}{a^{4}}}
$$

giving

$$
a(t)=a_{0} \sqrt{\cosh (\sigma t)}
$$

We have for this scale factor

$$
R=3 \sigma^{2}
$$

while $b$-s form an infinite sequence of polynomials of $\cosh (\sigma t)$.

One interesting observations is that the scale factor given by (3.3) is exactly the leading term in the series expansion

$$
H=\sigma \sqrt{1-\frac{a_{0}^{p}}{a^{p}}}
$$

in the limit $p \rightarrow 0$. Also note that for scale factor (3.3) we have

$$
\begin{aligned}
& R=12 \sigma^{2} t^{2}+6 \sigma, \square R=-72 \sigma^{3} t^{2}-24 \sigma, \ldots \\
& b_{0}=\sigma / 2, b_{1}=4 \sigma^{2} t^{2}, \ldots
\end{aligned}
$$

where we see explicitly that $R$ obeys a recursion relation while $b$ not.

In all these cases one need an infinite fine tuning as explained in the previous Subsection in order to perform summations in the equations of motion. Even though such a fine tuning may be not favorable the reformulation itself of equations of motion in terms of $a$ may be interesting and we are looking forward to go further in this direction. 
Note also that all the above configurations of the second type are solutions with $\mathcal{F}_{2}=0$.

\section{Configurations of the third type.}

The third type of configurations is extremely generic and the only known scale factor which makes us able to track equation up to some reasonable extent is

$$
a=a_{0} t^{p}
$$

for a generic $p$. For this scale factor we have

$$
R^{(l)}=\frac{r_{l}}{t^{2 l+2}}, \quad b_{l}=\frac{\beta_{l}}{t^{2 l+2}}
$$

where constant coefficients may be expressed through $p$ explicitly. Note that for odd $p$ the series for $R^{l}$ stops at a certain $l$ and the soluions falls back in the previous subclass when one recursion relation exists. Now it is a matter of a straightforward computation to see that one can satisfy the Einstein equations by imposing algebraic relations on coefficients $f_{1_{n}}$ and $f_{2_{n}}$. This is because internal summations over $l$ in expressions for $\mathcal{L}$-s do not involve the time variable $t$.

Note also that all the above solutions are still solutions with an arbitrary $\mathcal{F}_{4}$. Even though it is not important for the background one would change the perturbative picture playing with the $\mathcal{F}_{4}$ parameter.

\section{A road-map to perturbations}

Needless to say that even writing down the perturbation equations may be an unfeasible task in the above formulation of the model. Below in this Section we outline the general approach and emphasize what shall be developed shortly in subsequent papers since the full detailed analysis is expected to be rather cumbersome and deserves a separate paper.

\subsection{Bianchi identity}

We know that the Einstein equations are constrained with the Bianchi identity which says $\nabla_{\mu} G_{\nu}^{\mu} \equiv 0$. In our case we have more than just Einstein-Hilbert Lagrangian but all the additional ingredients we have are covariant terms. This guarantees that the Bianchi identity holds trivially without imposing any extra condition. On the other hand this implies thanks to arbitrariness of coefficients $f_{I_{n}}$ that each separate term does covariantly conserve. Indeed, each $f_{I_{n}}$ is a coefficient in front of some covariant structure in the Einstein equations, say $\tau_{\nu}^{\mu}$. Assuming that only one of $f$-s coefficients is non-zero we come to a conclusion that the corresponding structure must covariantly conserve due to Bianchi identities, i.e. $\nabla_{\mu} \tau_{\nu}^{\mu} \equiv$ 0 . In other words it resembles a conserving perfect fluid stress-energy tensor. The same argument is applicable to all the $f$-s coefficients as well as their arbitrary combinations. 
The above arguments imply that thanks to Bianchi identity the parts with different $\mathcal{F}_{I}$ covariantly conserve separately. To make use of this we define

$$
\begin{aligned}
T_{0}^{\mu} & =T_{\nu}^{\mu}, \\
T_{1}^{\mu} & =-2 \lambda \tilde{G}_{\nu}^{\mu} \tilde{\mathcal{F}}_{1}(\square) R+2 \lambda\left(\nabla^{\mu} \partial_{\nu}-\delta_{\nu}^{\mu} \square\right) \tilde{\mathcal{F}}_{1}(\square) R+\lambda \mathcal{L}_{1}^{\mu}-\frac{\lambda}{2} \delta_{\nu}^{\mu}\left(\mathcal{L}_{1}^{\sigma}+\overline{\mathcal{L}}_{1}\right), \\
T_{2}^{\mu} & =-\frac{1}{2} \lambda R \mathcal{F}_{2}(\square) \tilde{G}_{\nu}^{\mu}-2 \lambda \tilde{G}_{\beta}^{\mu} \mathcal{F}_{2}(\square) \tilde{G}_{\nu}^{\beta}+\frac{\lambda}{2} \delta_{\nu}^{\mu} \tilde{G}_{\beta}^{\alpha} \mathcal{F}_{2}(\square) \tilde{G}_{\alpha}^{\beta}+ \\
& +2 \lambda\left(\nabla_{\rho} \nabla_{\nu} \mathcal{F}_{2}(\square) \tilde{G}^{\mu \rho}-\frac{1}{2} \square \mathcal{F}_{2}(\square) \tilde{G}_{\nu}^{\mu}-\frac{1}{2} \delta_{\nu}^{\mu} \nabla_{\sigma} \nabla_{\rho} \mathcal{F}_{2}(\square) \tilde{G}^{\sigma \rho}\right)+ \\
& +\lambda \mathcal{L}_{2}^{\mu}-\frac{\lambda}{2} \delta_{\nu}^{\mu}\left(\mathcal{L}_{2 \sigma}^{\sigma}+\overline{\mathcal{L}}_{2}\right)+2 \lambda \tilde{\Delta}_{\nu}^{\mu}, \\
T_{4}^{\mu}{ }_{\nu} & =2 \lambda\left(R_{\alpha \beta}+2 \nabla_{\alpha} \nabla_{\beta}\right) \mathcal{F}_{4}(\square) C_{\nu}{ }^{\alpha \beta \mu} .
\end{aligned}
$$

Now Einstein equations can be written in an extremely concise form

$$
M_{P}^{2} G_{\nu}^{\mu}=\sum_{I} T_{I}^{\mu}-\Lambda \delta_{\nu}^{\mu}
$$

and moreover we have

$$
\nabla_{\mu} T_{I}^{\mu}=0 \text { for any } I .
$$

One recognizes here the system of minimally coupled perfect fluids minimally coupled to gravity. The perturbation technique is known in general (see $[32,40]$ for instance) but it is not obvious it can be applicable "as is" to our model as we will see shortly.

\subsection{Subtleties of the non-localities}

Having brought Einstein equations in our model to an already studied structure does not mean we are able easily, if at all, solve the perturbation equations.

It looks promising that we could clearly separate the whole equations into minimally coupled terms. But we have to stress here that each term is not a canonical perfect fluid, i.e. it cannot be written just in terms of energy density $\rho$, pressure density $p$ and four-fluid velocity $u_{\mu}$ as discussed in [41]. ${ }^{6}$ This results in presence of anisotropic stresses as well as entropic perturbations for our fluids at the level of perturbations. This in turn does not allow us to use the already known techniques straightforwardly since the system of perturbation equations (even non-local ones) will not be closed.

On the other hand all $T_{I}$ are not independent external stress energy tensors but rather some structures built on the metric. We therefore must be able in principle to have the corresponding perturbative quantities in terms of the metric perturbations.

Also one must not go straight with $T_{4}$ term since it represents a contribution which is absent in the background. It means, that energy and pressure for $T_{4}$ are both zero. Thus it is not obvious how one can define the equation of state parameter $w=p / \rho$ and the speed of sound $c_{s}^{2}=\dot{p} / \dot{\rho}$.

It is, however, the simplest term for performing actual calculations at the perturbative level. This is because it is zero on the background and completely traceless. The first point means that

$$
\delta T_{4}^{\mu}=2 \lambda\left(R_{\alpha \beta}+2 \nabla_{\alpha} \nabla_{\beta}\right) \mathcal{F}_{4}(\square) \delta C_{\nu}{ }^{\alpha \beta \mu}
$$

\footnotetext{
${ }^{6}$ In other words we cannot just have $T_{I}^{\mu}=(\rho+p) u^{\mu} u_{\nu}-p \delta_{\nu}^{\mu}$.
} 
The second point means that for isotropic perturbations

$$
\delta C_{\nu}{ }^{\alpha \beta \mu}=c(\eta) e^{i \vec{k} \vec{x}} P_{\nu}^{\alpha \beta \mu}
$$

where $\eta$ is the conformal time, $k$ is the comoving wave-vector and $P_{\nu}{ }^{\alpha \beta \mu}$ is a constant tensor which keeps all the symmetry properties of the Weyl tensor. We thus can use the same idea as in the previous Section for the traceless tensor $\tilde{G}_{\nu}^{\mu}$ and compute an action of some differential operator $\mathcal{D}$ on $\delta C_{\nu}{ }^{\alpha \beta \mu}$ as

$$
\mathcal{D} \delta C_{\nu}{ }^{\alpha \beta \mu}=(\tilde{\mathcal{D}} c(\eta)) e^{i \vec{k} \vec{x}} P_{\nu}{ }^{\alpha \beta \mu}
$$

with a simple algebraic relation between $\mathcal{D}$ and $\tilde{\mathcal{D}}$.

We can push these arguments further and draw some conclusions when $R$ or $\tilde{G}_{\nu}^{\mu}$ are perturbed in $T_{1}$ and $T_{2}$. We then are able to compute implicitly action of the non-local operators on those perturbed quantities. However, the real problem comes would one perturb the non-local operators themselves and compute the action of this perturbed operators on unperturbed background quantities. This is where presence of recursion relations is crucial. This was the key point why could we end up with final results in [18]. It is an open question how one would go in a general case when there are no recursion relations. We hope to address this issue in the forthcoming research [42].

We nevertheless can come up with a conclusion that the discussed above solution (3.2) is an example of a stable bounce configuration. Even though this particular solution creates recursion relations and in principle one would much easier write down system of perturbation equations we can claim the bounce phase is stable without tedious calculations. This stability is guaranteed because solution has analytic dependence on coefficients $f$. Stability of this solution with $\mathcal{F}_{2}=\mathcal{F}_{4}=0$ was explicitly demonstrated in [18]. Therefore turning on $\mathcal{F}_{2}$ and/or $\mathcal{F}_{4}$ must keep the configuration stable up to some range of coefficients $f_{2,4_{n}}$. Of course, this argument is not enough to find the allowed domain for new coefficients and this is the primary goal for the forthcoming paper [42].

\section{3 de Sitter limit}

The de Sitter limit is perhaps the most simple configuration for analysis of perturbations (apart from Minkowskian space-time, of course). The de Sitter Universe is described by the scale factor

$$
a=a_{0} e^{H t}
$$

and the corresponding Hubble function is just the constant $H$. This is where we see why the traceless tensor $\tilde{G}_{\nu}^{\mu}$ is convenient since it is identically zero in such a metric. Moreover the scalar curvature is a constant $R=12 H^{2}$. One can easily check this is a solution to our equations. But the most important that the part of equations which may be relevant for perturbations is much shorter since we can drop terms quadratic in $\tilde{G}_{\nu}^{\mu}$ as well as some others. Then the relevant equations become

$$
\begin{aligned}
& M_{P}^{2} \delta G_{\nu}^{\mu}=\delta T_{\nu}^{\mu}-2 \lambda \delta \tilde{G}_{\nu}^{\mu} \tilde{f}_{10} R+2 \lambda\left(\nabla^{\mu} \partial_{\nu}-\delta_{\nu}^{\mu} \square\right) \tilde{\mathcal{F}}_{1}(\square) \delta R-\frac{1}{2} \lambda R \mathcal{F}_{2}(\square) \delta \tilde{G}_{\nu}^{\mu}- \\
+ & 2 \lambda\left(\nabla_{\rho} \nabla_{\nu} \mathcal{F}_{2}(\square) \delta \tilde{G}^{\mu \rho}-\frac{1}{2} \square \mathcal{F}_{2}(\square) \delta \tilde{G}_{\nu}^{\mu}-\frac{1}{2} \delta_{\nu}^{\mu} \nabla_{\sigma} \nabla_{\rho} \mathcal{F}_{2}(\square) \delta \tilde{G}^{\sigma \rho}\right)- \\
- & \frac{\lambda}{2} \delta_{\nu}^{\mu} R\left(\tilde{\mathcal{F}}_{1}(\square)-\tilde{f}_{10}\right) \delta R+4 \lambda \nabla_{\alpha} \nabla_{\beta} \mathcal{F}_{4}(\square) \delta C_{\nu}{ }^{\alpha \beta \mu}
\end{aligned}
$$


with the trace

$$
-M_{P}^{2} \delta R=\delta T-6 \lambda \square \tilde{\mathcal{F}}_{1}(\square) \delta R-2 \lambda R\left(\tilde{\mathcal{F}}_{1}(\square)-\tilde{f}_{10}\right) \delta R-2 \lambda \nabla_{\rho} \nabla_{\mu} \mathcal{F}_{2}(\square) \delta \tilde{G}^{\mu \rho} .
$$

These can be analyzed up to the end explicitly for some configurations. To highlight the track towards solutions to the perturbation equations for scalar perturbations we note that $\delta R$ has an explicit expression in terms of Bardeen potentials $\Phi$ and $\Psi$, the two gauge invariant scalar degrees of freedom [43]. Then one can show that $i \neq j$ component of equation (5.2) is a non-local equation containing $\delta R$ and $\Phi-\Psi$, where $i, j$ are the spatial indexes. Assuming further that the matter is the radiation without anisotropic stresses and computing explicitly $\nabla_{\rho} \nabla_{\mu} \mathcal{F}_{2}(\square) \delta \tilde{G}^{\mu \rho}$ which is feasible thanks to the tracelessness of the $\tilde{G}^{\mu \rho}$ tensor we must end up with two non-local equations on $\Phi$ and $\Psi$.

Even though we can develop on this right away we are going to stop at this stage because there are many other serious and unrevealed questions regarding the de Sitter background in this type of models such as the ghost-free condition. These unexplored matters deserve a separate study which will include the detailed analysis of perturbations as well [42].

\section{Summary and Outlook}

We have considered in this paper the most general extension of GR based on inclusion of stringy motivated non-local operators and keeping the quadratic in curvature terms.

The primary goal was to find out an exact bouncing solution and it is for the first time as this task is accomplished up to the best of our knowledge. It is intriguing that the bouncing configuration is the cosine hyperbolic which is still a solution provided operator functions $\mathcal{F}_{2}$ and $\mathcal{F}_{4}$ which control the presence of extra terms are trivial. This indicates a presence of some symmetry one would have to find and so far is one of the open questions. A good point of our bouncing solution (3.2) is that it may be a solution in our model without extra matter which was not the case without $\mathcal{F}_{2}$ and $\mathcal{F}_{4}$. Also this solution requires just a couple of conditions which are very general and are easy to satisfy.

Moreover we have presented several other solutions which are not all bouncing solutions but anyway widen our understanding of the model. Practically we divided possible solutions into three groups based on the fact whether they create or not recursion relations when the scalar curvature and the Ricci tensor are being acted by powers of the d'Alembertian operator. Among all the found configurations the one which is (4.11) looks very interesting for the further study since it creates a bouncing Universe with the constant scalar curvature. It is a solution with $\mathcal{F}_{2}=0$ or for a very special $\mathcal{F}_{2}$ when all the coefficients are strictly fixed.

One more interesting point of this model is that the last term with the Weyl tensor squared in the Lagrangian does not contribute to the background at all. It, however, shows up in perturbations and it is interesting to see whether presence of this term can be efficiently used to tackle the problem of solving the perturbation equations.

Also we were able to bring the model formulation to such a form that was already analyzed at the perturbative level for other configurations. The perturbations can be analyzed in full at least numerically in the de Sitter limit and the clear way towards this was outlined. As it was mentioned in the main part of the paper, however, we postpone the more detailed and technical study of this question since it is more natural to join it with other unrevealed problems one can put for this model in the de Sitter space. Namely, ghost-free condition and subsequent quantization are the issues to be fully studied as well and results should appear shortly in [42]. 
The full analysis of perturbations is expected to be very hard in general. At the moment it is not even obvious one will be able to close the system of perturbation equations for scalar perturbations. Tensor perturbations may become simpler but still one would face the difficulty that effective anisotropic stresses present in the system. This is tough and open question at the moment.

Furthermore one question which we kept aside is the anisotropic perturbations during the contraction phase since this question is under investigation in the parallel project [44]. The generic expectation is that such perturbations must grow during the contraction phase and the main problem is to formulate the domains of parameters of the model allowing the bounce to happen smoothly. Approaches to study anisotropic perturbations in non-local as well as stringy-inspired models can be found in $[22,45]$ and references therein.

\section{Acknowledgments}

A.K. is supported by an "FWO-Vlaanderen" postdoctoral fellowship and also supported in part by Belgian Federal Science Policy Office through the Interuniversity Attraction Pole P7/37, the "FWO-Vlaanderen" through the project G.0114.10N and the RFBR grant 11-0100894.

\section{References}

[1] E. Komatsu et al. [WMAP Collaboration], Seven-Year Wilkinson Microwave Anisotropy Probe (WMAP) Observations: Cosmological Interpretation, Astrophys. J. Suppl. 192 (2011) 18 [arXiv:1001.4538 [astro-ph.CO]].

[2] A. Mazumdar and J. Rocher, Particle physics models of inflation and curvaton scenarios, Phys. Rept. 497 (2011) 85 [arXiv:1001.0993 [hep-ph]].

[3] A. Borde and A. Vilenkin, Eternal inflation and the initial singularity, Phys. Rev. Lett. 72 (1994) 3305 [gr-qc/9312022].

[4] A. D. Linde, D. A. Linde and A. Mezhlumian, From the Big Bang theory to the theory of a stationary universe, Phys. Rev. D 49 (1994) 1783 [gr-qc/9306035];

T. Vachaspati and M. Trodden, Causality and cosmic inflation, Phys. Rev. D 61 (1999) 023502 [gr-qc/9811037].

[5] A. Borde, A. H. Guth and A. Vilenkin, Inflationary space-times are incomplete in past directions, Phys. Rev. Lett. 90 (2003) 151301 [gr-qc/0110012].

[6] G. F. R. Ellis and R. Maartens, The emergent universe: Inflationary cosmology with no singularity, Class. Quant. Grav. 21 (2004) 223 [gr-qc/0211082];

G. F. R. Ellis, J. Murugan and C. G. Tsagas, The emergent universe: An explicit construction, Class. Quant. Grav. 21 (2004) 233 [gr-qc/0307112].

[7] S. W. Hawking and G. F. R. Ellis, The Large Scale Structure of Space-Time, Cambridge University, New York (1975).

[8] T. S. Koivisto, Cosmology of modified (but second order) gravity, AIP Conf. Proc. 1206 (2010) 79 [arXiv:0910.4097 [gr-qc]];

S. 'i. Nojiri and S. D. Odintsov, Unified cosmic history in modified gravity: from $F(R)$ theory to Lorentz non-invariant models, Phys. Rept. 505 (2011) 59 [arXiv:1011.0544 [gr-qc]];

S. Capozziello and V. Faraoni, Beyond Einstein Gravity: A Survey of Gravitational Theories for Cosmology and Astrophysics, Fund. Theor. Phys. 170 (2011), Springer, New York

[9] D. Lovelock, The Einstein tensor and its generalization, J. Math. Phys. 12 (1971) 498; 
D. Lovelock, Divergence-free tensorial concomitants, Aequationes Math. 4 (1970) 127;

D. Lovelock, Tensors differential forms and variational principles, Wiley-Interscience, New York, 1975.

[10] M. Ostrogradski, Memoires sur les equations differentielles relatives au probleme des isoperimetres, Mem. Ac. St. Petersburg VI 4 (1850) 385.

[11] T. Biswas, A. Mazumdar and W. Siegel, Bouncing universes in string-inspired gravity, JCAP 0603 (2006) 009 [hep-th/0508194].

[12] T. Biswas, T. Koivisto and A. Mazumdar, Towards a resolution of the cosmological singularity in non-local higher derivative theories of gravity, JCAP 1011 (2010) 008 [arXiv:1005.0590 [hep-th]].

[13] J. Khoury, Fading Gravity and Self-Inflation, Phys. Rev. D 76 (2007) 123513 [hep-th/0612052]; P. Stephens, Inflation from a Non-Local Theory of Gravity, arXiv:0908.2787 [gr-qc]; G. Calcagni and G. Nardelli, Non-local gravity and the diffusion equation, Phys. Rev. D 82 (2010) 123518 [arXiv:1004.5144 [hep-th]].

[14] T. Biswas, R. Brandenberger, A. Mazumdar and W. Siegel, Non-perturbative gravity, Hagedorn bounce and CMB, JCAP 0712 (2007) 011 [hep-th/0610274].

[15] J. W. Moffat, Ultraviolet Complete Quantum Gravity, Eur. Phys. J. Plus 126 (2011) 43 [arXiv:1008.2482 [gr-qc]];

L. Modesto, Super-renormalizable Quantum Gravity, arXiv:1107.2403 [hep-th];

L. Modesto, Towards a finite quantum supergravity, arXiv:1206.2648 [hep-th];

L. Modesto, Super-renormalizable Higher-Derivative Quantum Gravity, arXiv:1202.0008

[hep-th];

F. Briscese, A. Marciano, L. Modesto and E. N. Saridakis, Inflation in (Super-)renormalizable Gravity, arXiv:1212.3611 [hep-th];

K. Krasnov, Renormalizable Non-Metric Quantum Gravity?, hep-th/0611182;

K. Krasnov, Non-Metric Gravity I: Field Equations, Class. Quant. Grav. 25 (2008) 025001 [gr-qc/0703002];

K. Krasnov and Yu. Shtanov, Non-Metric Gravity II: Spherically Symmetric Solution, Missing Mass and Redshifts of Quasars, Class. Quant. Grav. 25 (2008) 025002 [arXiv:0705.2047 [gr-qc]]; I. P. Neupane, Localized gravity with higher curvature terms, Class. Quant. Grav. 19 (2002) 5507 [hep-th/0106100];

I. P. Neupane, Consistency of higher derivative gravity in the brane background, JHEP 0009 (2000) 040 [hep-th/0008190].

[16] N. Arkani-Hamed, S. Dimopoulos, G. Dvali and G. Gabadadze, Non-local modification of gravity and the cosmological constant problem, hep-th/0209227.

[17] A.O. Barvinsky, Nonlocal action for long-distance modifications of gravity theory, Phys. Lett. B 572 (2003) 109 [hep-th/0304229].

[18] T. Biswas, A. S. Koshelev, A. Mazumdar and S. Y. .Vernov, Stable bounce and inflation in non-local higher derivative cosmology, JCAP 1208 (2012) 024 [arXiv:1206.6374 [astro-ph.CO]].

[19] T. Biswas, E. Gerwick, T. Koivisto and A. Mazumdar, Towards singularity and ghost free theories of gravity, Phys. Rev. Lett. 108 (2012) 031101 [arXiv:1110.5249 [gr-qc]].

[20] T. Biswas, T. Koivisto and A. Mazumdar, Nonlocal theories of gravity: the flat space propagator, arXiv:1302.0532 [gr-qc].

[21] S. Deser and R. P. Woodard, Nonlocal Cosmology, Phys. Rev. Lett. 99 (2007) 111301 [arXiv:0706.2151 [astro-ph]];

S. 'i. Nojiri and S. D. Odintsov, Modified non-local- $F(R)$ gravity as the key for the inflation and dark energy, Phys. Lett. B 659 (2008) 821 [arXiv:0708.0924 [hep-th]]; 
S. Jhingan, S. Nojiri, S. D. Odintsov, M. Sami, IThongkool and S. Zerbini, Phantom and non-phantom dark energy: The Cosmological relevance of non-locally corrected gravity, Phys. Lett. B 663 (2008) 424 [arXiv:0803.2613 [hep-th]];

T. Koivisto, Dynamics of Nonlocal Cosmology, Phys. Rev. D 77 (2008) 123513

[arXiv:0803.3399 [gr-qc]];

T. S. Koivisto, Newtonian limit of nonlocal cosmology, Phys. Rev. D 78 (2008) 123505

[arXiv:0807.3778 [gr-qc]];

C. Deffayet and R. P. Woodard, Reconstructing the Distortion Function for Nonlocal

Cosmology, JCAP 0908 (2009) 023 [arXiv:0904.0961 [gr-qc]];

K. A. Bronnikov and E. Elizalde, Spherical systems in models of nonlocally corrected gravity,

Phys. Rev. D 81 (2010) 044032 [arXiv:0910.3929 [hep-th]];

J. Kluson, Non-Local Gravity from Hamiltonian Point of View, JHEP 1109 (2011) 001

[arXiv:1105.6056 [hep-th]];

S. 'i. Nojiri, S. D. Odintsov, M. Sasaki and Y. -l. Zhang, Screening of cosmological constant in non-local gravity, Phys. Lett. B 696 (2011) 278 [arXiv:1010.5375 [gr-qc]];

Y. -l. Zhang and M. Sasaki, Screening of cosmological constant in non-local cosmology, Int. J.

Mod. Phys. D 21 (2012) 1250006 [arXiv:1108.2112 [gr-qc]];

A. O. Barvinsky, Serendipitous discoveries in nonlocal gravity theory, Phys. Rev. D 85 (2012)

104018 [arXiv:1112.4340 [hep-th]];

M. G. Romania, N. C. Tsamis and R. P. Woodard, Quantum Gravity and Inflation, arXiv:1204.6558 [gr-qc].

[22] E. Elizalde, E. O. Pozdeeva and S. Yu. Vernov, De Sitter Universe in Non-local Gravity, Phys. Rev. D 85 (2012) 044002 [arXiv:1110.5806 [astro-ph.CO]].

[23] P. G. O. Freund and M. Olson, Nonarchimedean Strings, Phys. Lett. B 199 (1987) 186;

P. G. O. Freund and E. Witten, Adelic String Amplitudes, Phys. Lett. B 199 (1987) 191;

L. Brekke, P. G. O. Freund, M. Olson and E. Witten, Nonarchimedean String Dynamics, Nucl. Phys. B 302 (1988) 365;

P. H. Frampton, Ya. Okada, Effective Scalar Field Theory of P-Adic String, Phys. Rev. D 37 (1988) 3077;

V.S. Vladimirov, I.V. Volovich, I.V. Zelenov, p-adic Analysis and Mathematical Physics,

Singapore: World Scientific (1994);

B. Dragovich, A. Yu. Khrennikov, S. V. Kozyrev and I. V. Volovich, On p-Adic Mathematical Physics, Anal. Appl. 1 (2009) 1 [arXiv:0904.4205 [math-ph]].

[24] B. Dragovich, Zeta Strings, hep-th/0703008;

B. Dragovich, Zeta Nonlocal Scalar Fields, Theor. Math. Phys. 157 (2008) 1671

[arXiv:0804.4114 [hep-th]].

[25] M. R. Douglas and S. H. Shenker, 'Strings in Less Than One-Dimension, Nucl. Phys. B 335 (1990) 635.

[26] T. Biswas, M. Grisaru and W. Siegel, Linear Regge Trajectories from Worldsheet Lattice Parton Field Theory, Nucl. Phys. B 708 (2005) 317 [hep-th/0409089].

[27] V. A. Kostelecky and S. Samuel, On a Nonperturbative Vacuum for the Open Bosonic String, Nucl. Phys. B 336 (1990) 263;

I. Ya. Aref'eva, L. V. Joukovskaya and A. S. Koshelev, Time evolution in superstring field theory on non-BPS brane. I: Rolling tachyon and energy-momentum conservation, JHEP 0309 (2003) 012 [hep-th/0301137];

G. Calcagni and G. Nardelli, Tachyon Solutions in Boundary and Cubic String Field Theory, Phys. Rev. D 78 (2008) 126010 [arXiv:0708.0366[hep-th]].

[28] E. Witten, Noncommutative Geometry and String Field Theory, Nucl. Phys. B 268 (186) 253;

A. Sen, Rolling tachyon, JHEP 0204 (2002) 048 [hep-th/0203211];

A. Sen, Tachyon matter, JHEP 0207 (2002) 065 [hep-th/0203265]; 
I. Ya. Aref'eva, D.M. Belov, A.A. Giryavets, A.S. Koshelev, P.B. Medvedev, Noncommutative Field Theories and (Super)String Field Theories, hep-th/0111208;

W. Taylor and B. Zwiebach, D-branes, Tachyons, and String Field Theory, [hep-th/0311017].

[29] I. Ya. Aref'eva, Nonlocal string tachyon as a model for cosmological dark energy, AIP Conf. Proc. 826 (2006) 301 [astro-ph/0410443];

G. Calcagni, Cosmological tachyon from cubic string field theory, JHEP 0605 (2006) 012 [hep-th/0512259];

I. Ya. Aref'eva, L. V. Joukovskaya and S. Yu. Vernov, Bouncing and accelerating solutions in nonlocal stringy models, JHEP 0707 (2007) 087 [hep-th/0701184];

J. E. Lidsey, Stretching the inflaton potential with kinetic energy, Phys. Rev. D 76 (2007) 043511 [hep-th/0703007];

G. Calcagni, M. Montobbio and G. Nardelli, A Route to nonlocal cosmology, Phys. Rev. D 76 (2007) 126001 [arXiv:0705.3043 [hep-th]];

N. Barnaby, T. Biswas and J. M. Cline, p-adic Inflation, JHEP 0704 (2007) 056 [hep-th/0612230];

N. Barnaby and J. M. Cline, Large Nongaussianity from Nonlocal Inflation, JCAP 0707 (2007) 017 [arXiv:0704.3426 [hep-th]];

I. Ya. Aref'eva, L. V. Joukovskaya and S. Yu. Vernov, Dynamics in nonlocal linear models in the Friedmann-Robertson-Walker metric, J. Phys. A 41 (2008) 304003 [arXiv:0711.1364 [hep-th]];

D. J. Mulryne and N. J. Nunes, Diffusing non-local inflation: Solving the field equations as an initial value problem, Phys. Rev. D 78 (2008) 063519 [arXiv:0805.0449 [hep-th]];

G. Calcagni and G. Nardelli, Nonlocal instantons and solitons in string models, Phys. Lett. B 669 (2008) 102 [arXiv:0802.4395 [hep-th]];

L. Joukovskaya, Dynamics in nonlocal cosmological models derived from string field theory, Phys. Rev. D 76 (2007) 105007 [arXiv:0707.1545 [hep-th]];

N. Barnaby and N. Kamran, Dynamics with infinitely many derivatives: The Initial value problem, JHEP 0802 (2008) 008 [arXiv:0709.3968 [hep-th]];

I. Ya. Aref'eva and A. S. Koshelev, Cosmological Signature of Tachyon Condensation, JHEP 0809 (2008) 068 [arXiv:0804.3570 [hep-th]];

S. Yu. Vernov, Localization of the SFT inspired Nonlocal Linear Models and Exact Solutions, Phys. Part. Nucl. Lett. 8 (2011) 310 [arXiv:1005.0372 [astro-ph.CO]];

N. Barnaby, A New Formulation of the Initial Value Problem for Nonlocal Theories, Nucl. Phys. B 845 (2011) 1 [arXiv:1005.2945 [hep-th]];

F. Galli, A.S. Koshelev, Perturbative stability of SFT-based cosmological models, JCAP 1105 (2011) 012 [arXiv:1011.5672 [hep-th]].

[30] A. S. Koshelev, Non-local SFT Tachyon and Cosmology, JHEP 0704 (2007) 029 [hep-th/0701103];

N. Barnaby and N. Kamran, Dynamics with Infinitely Many Derivatives: Variable Coefficient Equations, JHEP 0812 (2008) 022 [arXiv:0809.4513 [hep-th]];

G. Calcagni, M. Montobbio and G. Nardelli, Localization of nonlocal theories, Phys. Lett. B 662 (2008) 285 [arXiv:0712.2237 [hep-th]].

[31] S. Yu. Vernov, Localization of Non-local Cosmological Models with Quadratic Potentials in the case of Double Roots, Class. Quant. Grav. 27 (2010) 035006 [arXiv:0907.0468 [hep-th]].

[32] A. S. Koshelev and S. Yu. Vernov, Cosmological perturbations in SFT inspired non-local scalar field models, arXiv:0903.5176 [hep-th];

A. S. Koshelev and S. Yu. Vernov, Analysis of scalar perturbations in cosmological models with a non-local scalar field, Class. Quant. Grav. 28 (2011) 085019 [arXiv:1009.0746 [hep-th]].

[33] H. J. Blome, C. Chicone, F. W. Hehl and B. Mashhoon, Nonlocal Modification of Newtonian Gravity, Phys. Rev. D 81 (2010) 065020 [arXiv:1002.1425 [gr-qc]];

S. Nesseris and A. Mazumdar, Newton's constant in $f\left(R, R_{\mu \nu} R^{\mu \nu}, \square R\right)$ theories of gravity and 
constraints from BBN, Phys. Rev. D 79 (2009) 104006 [arXiv:0902.1185 [astro-ph.CO]]; B. Mashhoon, Toward a nonlocal theory of gravitation, Annalen Phys. 16 (2007) 57 [hep-th/0608010].

[34] T. Biswas, J. A. R. Cembranos and J. I. Kapusta, Thermal Duality and Hagedorn Transition from p-adic Strings, Phys. Rev. Lett. 104 (2010) 021601 [arXiv:0910.2274 [hep-th]]

T. Biswas, J. A. R. Cembranos and J. I. Kapusta, Finite Temperature Solitons in Non-Local Field Theories from p-Adic Strings, Phys. Rev. D 82 (2010) 085028 [arXiv:1006.4098 [hep-th]]; T. Biswas, J. A. R. Cembranos and J. I. Kapusta, Thermodynamics and Cosmological Constant of Non-Local Field Theories from p-Adic Strings, JHEP 1010 (2010) 048 [arXiv:1005.0430 [hep-th]].

[35] T. Biswas, J. Kapusta and A. Reddy, String Field Theory at Finite Temperature, arXiv:1201.1580 [hep-th].

[36] A. O. Barvinsky and Y. .V. Gusev, New representation of the nonlocal ghost-free gravity theory, arXiv:1209.3062 [hep-th].

[37] A. S. Koshelev and S. Yu. Vernov, On bouncing solutions in non-local gravity, Phys. Part. Nuclei 43 (2012) 666 [arXiv:1202.1289 [hep-th]].

[38] I. Dimitrijevic, B. Dragovich, J. Grujic and Z. Rakic, On Modified Gravity, arXiv:1202.2352 [hep-th].

[39] I.S. Gradshteyn, I.M. Ryzhik, Table of Integrals, Series, and Products, edited by A. Jeffrey and D. Zwillinger, Academic Press, New York, 7th edition (2007).

[40] J. -c. Hwang and H. Noh, Cosmological perturbations with multiple fluids and fields, Class. Quant. Grav. 19 (2002) 527 [astro-ph/0103244].

[41] V.F. Mukhanov, H.A. Feldman, and R.H. Brandenberger, Theory of Cosmological Perturbations, Phys. Rep. 215 (1992) 203.

[42] A.S. Koshelev, work in progress

[43] J.M. Bardeen, Gauge Invariant Cosmological Perturbations, Phys. Rev. D 22 (1980) 1882.

[44] A.S. Koshelev, S.V. Vernov, work in progress

[45] I. Ya. Aref'eva, N. V. Bulatov, L. V. Joukovskaya and S. Yu. Vernov, The NEC Violation and Classical Stability in the Bianchi I Metric, Phys. Rev. D 80 (2009) 083532 [arXiv:0903.5264 [hep-th]]. 